\title{
Ultra-low timing-jitter passively mode- locked fiber lasers for long-distance timing synchronization
}

F. Ö. Ilday

A,. Winter

J.-W. Kim

J. Chen

P. Schmüser

H. Schlarb

F. X. Kärtner 


\title{
Ultra-low timing-jitter passively mode-locked fiber lasers for long-distance timing synchronization
}

\author{
F. Ö. Ilday ${ }^{a, b}$, A. Winter ${ }^{c, d}$, J.-W. Kim ${ }^{b}$, J. Chen $^{b}$, P. Schmüser ${ }^{c}$, H. Schlarb ${ }^{d}$, and F. X. \\ Kärtner $^{b}$ \\ ${ }^{a}$ Bilkent Üniversitesi, Ankara 06800, Turkey; \\ ${ }^{b}$ Massachusetts Institute of Technology, Cambridge, MA, USA; \\ ${ }^{c}$ Universität Hamburg, Hamburg, Germany; \\ ${ }^{d}$ Deutsches Elektronen-Synchrotron, Hamburg, Germany
}

\begin{abstract}
One of the key challenges for the next-generation light sources such as X-FELs is to implement a timing stabilization and distribution system to enable $\sim 10$ fs synchronization of the different RF and laser sources distributed in such facilities with distances up to a few kilometers. These requirements appear to be beyond the capability of traditional RF distribution systems based on temperature-stabilized coaxial cables. A promising alternative is to use an optical transmission system: A train of pulses generated from a laser with low timing jitter is distributed over length-stabilized fiber links to remote locations. The repetition frequency of the pulse train and its higher harmonics contain the synchronization information. At the remote locations, RF signals are extracted simply by using a photodiode and a suitable bandpass filter to pick the desired harmonic of the laser repetition rate. Passively mode-locked Er-doped fiber lasers provide excellent long-term stability. The laser must have extremely low timing jitter, particularly at high frequencies $(>1 \mathrm{kHz})$. Ultimately, the timing jitter is limited by quantum fluctuations in the number of photons making up the pulse and the incoherent photons added in the cavity due to spontaneous emission. The amplitude and phase noise of a home-built laser, generating 100-fs, 1-nJ pulses, was characterized. The measured phase noise (timing jitter) is sub-10 fs, from $1 \mathrm{kHz}$ to Nyquist frequency. In addition to synchronization of accelerators, the ultra-low timing jitter pulse source can find applications in next-generation telecommunication systems.
\end{abstract}

Keywords: ultrafast optics, photonics, fiber, laser, RF, phase-noise, timing jitter

\section{INTRODUCTION}

One of the key challenges for the new fourth generation light sources such as the XFEL is to implement a timing stabilization and distribution system that allows the full exploitation of the potentially $\sim 10$ fs $\mathrm{x}$-ray pulse for time-resolved studies. To this end, an ultra-stable timing and synchronization system must be implemented, covering the critical subsystems of the machine and the experimental area, which are spread over distances as large as several kilometers.

The electron beam needs to enter the undulator with timing jitter comparable to the pulse duration, which puts significant pressure on the synchronization system of the XFEL and requires point-to-point stabilization of various RF frequencies for the critical components (booster section, injector, bunch compressors and experimental area) with femtosecond precision. This translates to an amplitude and phase stability of the RF in the critical cavities of $10^{-4}$ and $0.01 \mathrm{deg}$ respectively. The required amplitude stability levels have already been achieved in present day facilites, e.g. at JLAB and the DESY VUV-FEL. ${ }^{1,2}$ The best phase stability reported for superconducting cavities is on the order of 0.03 degrees; improving this to the $0.01 \mathrm{deg}$ level (21 fs at 1.3 $\mathrm{GHz}$ ) seems feasible. However, in order to accurately measure the phase stability, one requires a high-quality reference with much smaller phase jitter than the signal to be measured. A key challenge is to provide this reference in facilities spanning a few kilometers in length.

E-mail: ilday@bilkent.edu.tr

Active and Passive Optical Components for Communications VI edited by Achyut K. Dutta, Yasutake Ohishi, Niloy K. Dutta, Jesper Moerk Proc. of SPIE Vol. 6389, 63890L, (2006) · 0277-786X/06/\$15 - doi: 10.1117/12.687364

Proc. of SPIE Vol. 6389 63890L-1 


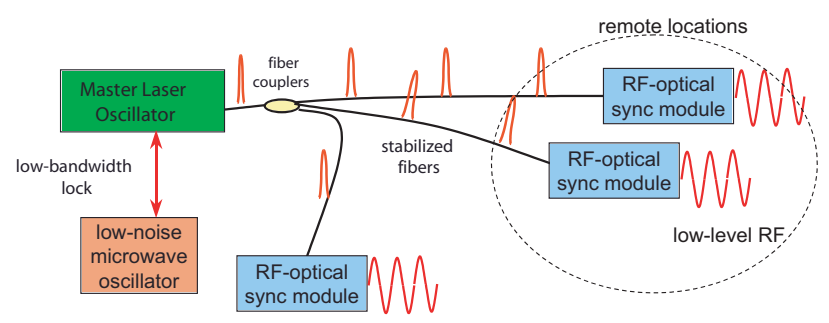

Figure 1. Schematic of the optical timing synchronization system.

These requirements on the timing stability appear to be beyond the capability of traditional RF distribution systems based on temperature-stabilized coaxial cables. A promising way to reach this goal is by using an optical transmission system, ${ }^{3}$ depicted schematically in Figure $1 .{ }^{4}$ A train of sub-picosecond pulses of light generated from a mode-locked laser with very low timing jitter is distributed over actively length-stabilized optical fiber links to an arbitrary number of remote locations. The precise repetition frequency of the pulse train, as well as its upper harmonics, contain the synchronization information. At the remote locations, low-level RF signals can be extracted simply by using a photodiode and a suitable bandpass filter to pick the desired harmonic of the laser repetition rate, or by phase locking an RF source to a harmonic of the pulse train. ${ }^{5}$

\section{TIMING STABILITY REQUIREMENTS FOR THE X-RAY PULSES}

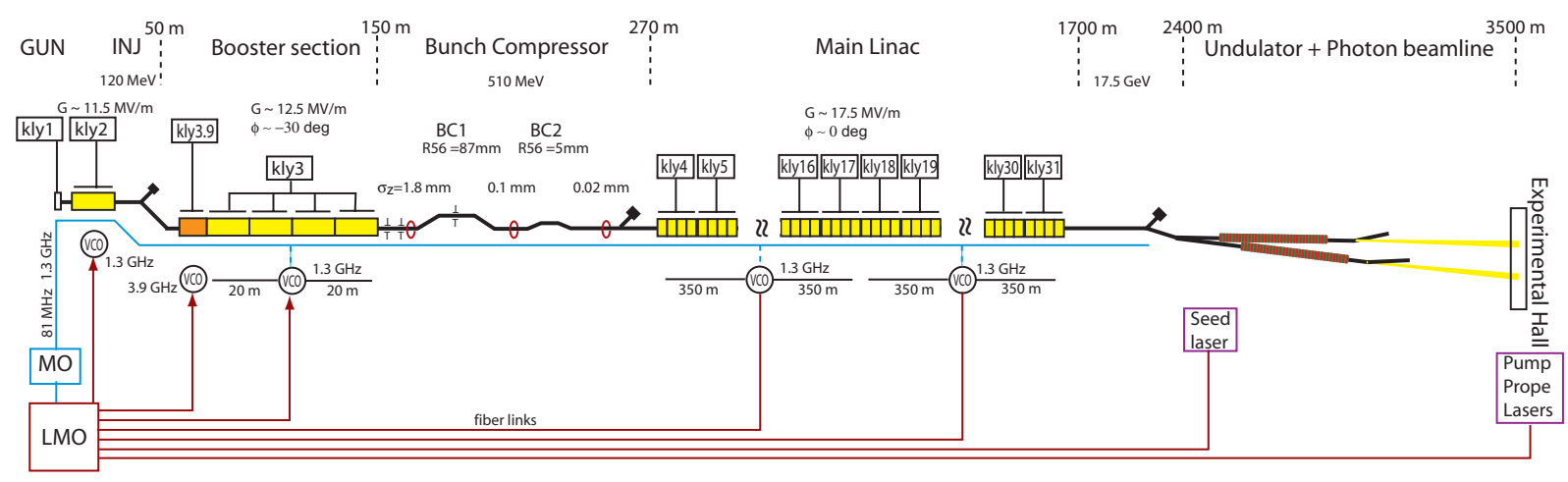

Figure 2. Layout of a 4th generation light source.

The layout of the European XFEL is shown in Figure 2 as an example of a fourth generation light source. The most critical sections of the machine in terms of inducing timing jitter onto the electron bunch are the injector and booster sections. Here, the electron bunch is accelerated off-crest to induce a chirp to make bunch compression possible. If amplitude and phase of the RF inside the cavities varies, this translates to a change in the centroid energy of the electron bunch, which is in turn directly converted into timing jitter by the bunch compressor chicane. A timing jitter on the order of the desired x-ray pulse width requires amplitude and phase stability of $10^{-4}$ and $0.01 \mathrm{deg}$ respectively. This condition is relaxed by an order of magnitude in the main drive linac, as the on-crest acceleration makes the electron bunch less susceptible to amplitude and phase errors. If a seeding option is considered requiring an external laser system, as proposed for e.g. the FERMI facility, ${ }^{6}$ the timing jitter between the seed laser pulse and the electron bunch becomes a crucial issue for the quality of the x-ray pulse generated. Ideally, the seed laser pulse should have a flat-top profile and its pulse-width should be comparable to the electron bunch length. If a large timing jitter is present, the efficiency will drop dramatically and this timing jitter will directly transfer onto the final x-ray pulse. The seed laser is one of the components of the accelerator requiring the most stable reference and tightest lock possible. A similar argument is true for the probe laser systems in the experimental hall. To fully exploit the potential of a $\sim 10$ 
fs long x-ray pulse, the timing jitter between the probe pulse and the x-ray pulse must be kept to a minimum. Single-shot arrival time measurements might make it possible to sort the data taken afterwards according to the arrival time of the electron bunch, but a reliable arrival time measurement with 10 fs resolution has not been shown to date. Furthermore, the time it takes to accumulate a full data set increases drastically as a result of timing jitter between pump and probe pulses. Therefore, the probe laser systems also require a tight synchronization to the accelerator reference frequency, which makes a the stable distribution of that reference frequency to the experimental hall a key challenge. ${ }^{7}$ A possible solution is direct seeding of fiber amplifiers and Ti:Sapphire-based amplifiers with the optical pulses, distributing the timing information, or their second harmonics, respectively. This scenario has the inherent advantage that no timing jitter is added due to the generation of the seed pulse. Additional jitter imparted during the amplification process must be minimized.

\section{MODE-LOCKED FIBER LASERS}

Mode-locked fiber lasers are a natural choice to realize an optical master oscillator, because of the ease of coupling to the fiber distribution system, their excellent long-term stability, and the well-developed and mature component base available at the optical communications wavelength of $1550 \mathrm{~nm}$. Recently, their technical capabilities have also improved significantly. ${ }^{8,9} \mathrm{Yb}$-doped and Er-doped fiber lasers offer stable and practical platforms for short pulse generation, at $1 \mu \mathrm{m}$ and $1.5 \mu \mathrm{m}$, respectively. Fiber lasers can generate pulses from picosecond down to 35 fs in duration by simultaneous phase coherent lasing of multiple longitudinal modes spaced in frequency by the pulse repetition rate of the laser. During photodetection, these optical modes beat in the photodetector and generate all harmonics of the repetition rate within the bandwidth of the photodetector.

Mode-locking is initiated by a mechanism providing lower loss (hence, higher net gain) for a pulse than for $\mathrm{cw}$ radiation, leading to pulse formation from intra-cavity noise as soon as the laser is turned on. In the case of active mode-locking, this is a high-speed modulator. For passively mode-locked lasers, this is achieved by a real or artificial saturable absorber. For brevity, we restrict the following description to passive mode-locking. Once the pulses are shortened, the laser dynamics are dominated by an interplay of group velocity dispersion (different frequencies have different speeds) and Kerr nonlinearity (the refractive index depends on intensity), leading to the formation of soliton-like pulses, which intrinsically balance dispersion and nonlinearity. ${ }^{10}$ As the gain has a finite bandwidth, the generated pulses need to be stabilized by the saturable absorber, which favors the pulse and suppresses any cw-radiation. At the simplest level, short-pulse laser dynamics can be characterized by four processes: gain, saturable absorber, Kerr nonlinearity, and dispersion interacting in a periodic structure, defined by the physical cavity (Fig. 3a).

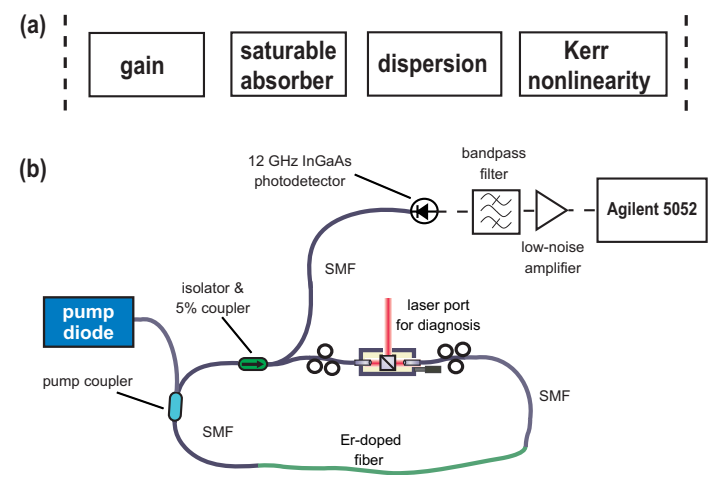

Figure 3. (a) The four effects governing spulse shaping in mode-locked lasers. (b) Schematic of the experimental setup: SMF, single-mode fiber.

In fiber lasers, the fiber assumes multiple roles, providing nonlinear and dispersive effects that dictate the soliton-like pulse shaping mechanism and moreover shielding against fast environmental fluctuations. The Eror Yb-doped fiber segments form the gain medium, pumped conveniently by low-cost, fiber-coupled $980 \mathrm{~nm}$ diode lasers. A representative schematic of the laser is presented in Figure 3b, where saturable absorption is implemented by nonlinear polarization rotation in the fiber. 


\section{NOISE PERFORMANCE OF MODE-LOCKED FIBER LASERS}

It is essential that the laser serving as the master oscillator has extremely low timing jitter, particularly at high frequencies $(>10 \mathrm{kHz})$, where further suppression through feedback is difficult. The timing of the pulse circulating in the laser cavity is affected by the intrinsic noise sources such as pump noise and amplified spontaneous emission noise from the amplification process. Ultimately, the timing jitter is limited by quantum fluctuations in the number of photons making up the pulse and the incoherent photons added in the cavity due to spontaneous emission.

The noise characteristics of mode-locked lasers is well-described using soliton-perturbation theory, along with quantum noise sources. ${ }^{11,12}$ These perturbations cause fluctuations in amplitude, phase, timing and center frequency. The last of these further contributes to timing in the presence of dispersion, i.e. a shift in center frequency is translated into timing shift via dispersion, which is known as the Gordon-Haus effect. ${ }^{13}$ For a fiber laser with small net dispersion and otherwise typical parameters, the quantum-limit is extremely small, in the other of $1 \mathrm{fs}$ (from $1 \mathrm{kHz}$ to $25 \mathrm{MHz}$, for a repetition rate of $50 \mathrm{MHz}$ ).

The noise performance of both an Er-doped fiber laser (EDFL) and an Yb-doped fiber laser (YDFL) were characterized. The EDFL is a stretched-pulse laser, implementing dispersion management ${ }^{14}$ producing pulses compressible down to $100 \mathrm{fs}$ and $1 \mathrm{~nJ}$ of energy at a repetition rate of $40 \mathrm{MHz}$, centered at $1550 \mathrm{~nm}$. A schematic of the EDFL and the experimental setup is shown in Figure 3. The YDFL is configured to produce pulses compressible down to 70 fs with $2 \mathrm{~nJ}$ energy content, with a repetition rate of $36 \mathrm{MHz}$, centered at 1030 nm. ${ }^{8}$ The YDFL (not shown) has a cavity similar to that of the EDFL. Both of the lasers are free-running, in the sense that the cavity length is uncontrolled and subject to slow, thermally induced fluctuations.

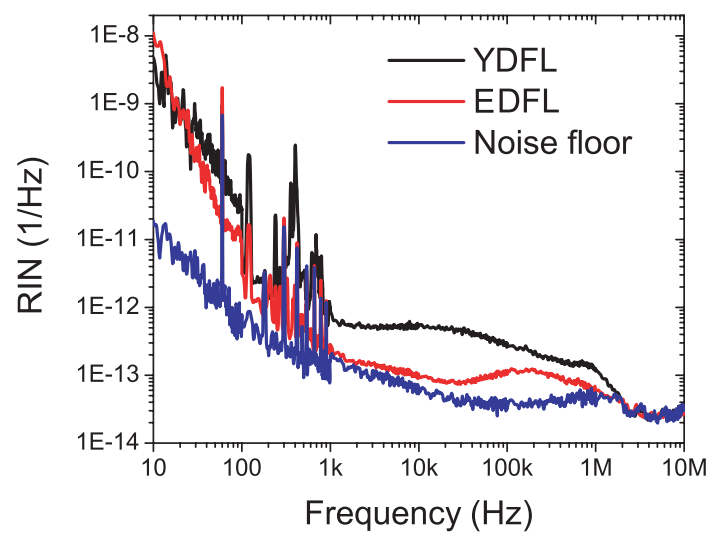

Figure 4. Relative intensity noise (RIN) of the EDFL and YDFL, along with the measurement noise floor.

The amplitude noise of the fiber lasers has been characterized. Figure 4 shows the relative intensity noise (RIN) of both lasers from $10 \mathrm{~Hz}$ to $1 \mathrm{MHz}$. The EDFL shows slightly lower high-frequency noise than the YDFL, which may be due to the different amplifier media and pulse shaping processes at work. The integrated RIN measured from $10 \mathrm{kHz}$ to $1 \mathrm{MHz}$ is about $0.04 \% \mathrm{rms}$ for the YDFL, and $0.03 \% \mathrm{rms}$ for the EDFL, compared to the average power level. Figure 5 shows the single sideband phase noise spectrum of the harmonic at $1.3 \mathrm{GHz}$ extracted from the pulse train upon photodetection and filtering. This phase noise spectrum can be converted into a timing jitter using $\Delta t=\frac{\sqrt{2 \int L\left(f^{\prime}\right) d f^{\prime}}}{2 \pi f_{0}}$. The integrated timing jitter from $1 \mathrm{kHz}$ to the respective Nyquist bandwidths, i.e., half of the laser repetition rate, is measured to be about $18 \mathrm{fs}$ and $10 \mathrm{fs}$ for the YDFL and EDFL, respectively. For comparison, the phase noise of a very low noise frequency generator, a Marconi 2041, is also plotted. As the lasers are free running, the performance of the microwave oscillator is slightly superior in the low frequency regime $(<10 \mathrm{kHz})$, but at frequencies of $\sim 100 \mathrm{kHz}$, the mode locked lasers reach a comparable level of stability, with the EDFL having the lowest noise among the three at frequencies higher than $20 \mathrm{kHz}$. Both lasers would be already suitable for an overall sub-100 fs timing distribution system, which is an important next step to achieve in several FEL facilities. On the long run, the EDFL seems to be a 
stronger candidate for a master oscillator due to the availability of a larger variety of components at $1550 \mathrm{~nm}$, and perhaps more importantly, transmission fibers with both signs of dispersion, which allow the construction of dispersion-compensated fiber links.

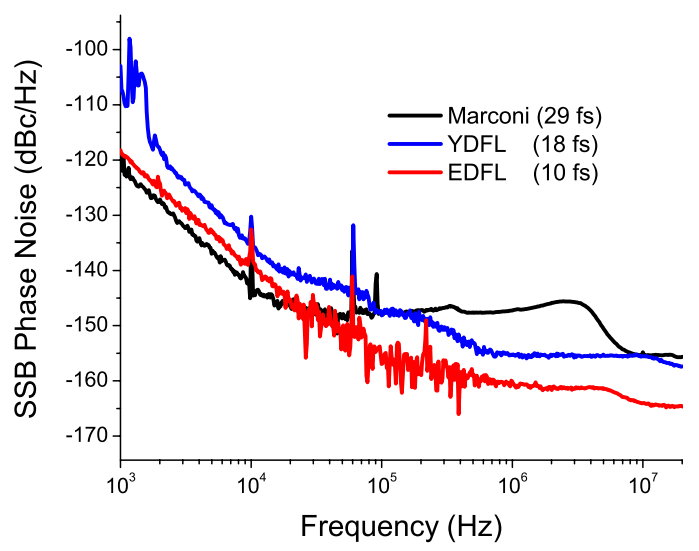

Figure 5. Single-sideband phase noise spectral density for the EDFL, YDFL, and a Marconi 2041 signal generator.

The measured timing jitter for both lasers is substantially higher than the noise limit given by the spontaneous emission noise. Several effects in the photodetection process lead to significant distortion of the actual phase noise spectrum. Due to the filtering of a single harmonic of the laser repetition rate, the power level of this harmonic is on the order of $-20 \mathrm{dBm}$, even when saturating a typical photodetector. The thermal noise floor of the $50 \mathrm{Ohm}$ resistor used to terminate the photodiode is at $-178 \mathrm{dBm}$. That results in a minimum noise floor of $-158 \mathrm{dBc}$ for the single sideband phase noise. The phase noise measurement device employed in this study (Agilent 5052) implements a correlation technique, which provides an additional noise suppression of up to $10 \mathrm{~dB}$. Even with this improvement, the phase noise of the reference oscillator itself constitutes another measurement limit. Another effect, which plagues phase noise measurements, is amplitude to phase conversion in the photodiode. ${ }^{15}$ The EDFL and YDFL have extremely low intensity fluctuations, nevertheless amplitude to phase conversion may contribute as much as 5 fs additional timing jitter.

\section{MEASUREMENTS IN AN ACCELERATOR ENVIRONMENT}

In order to verify that laboratory performance can be transfered to an accelerator environment without degredation, measurements were conducted at the MIT-Bates Linear Accelerator Center. We utilized a 500 m-long single-mode optical fiber link, which was already installed to achieve picosecond-stability optical signal transmission. The experiment consisted of three seperate parts:

(i) Locking of the EDFL to the S-band master oscillator at the Bates Facility to reduce the close-in noise of the laser system,

(ii) Stabilizing the fiber link with a RF-based feedback to reduce the timing jitter added by the transmission to a few femtoseconds,

(iii) Recovering a returning RF signal after $1 \mathrm{~km}$ of total travel through the fiber link with minimal added jitter.

The entire experiment was conducted over a time span of 3 weeks. The fiber laser worked reliably during this time without loss of mode-locking or significant increase of its phase noise.

\subsection{LOCKING OF THE EDFL TO THE S-BAND REFERENCE}

A schematic of the experiment is shown in Figure 6. The EDFL runs at a repetition rate of $40.22 \mathrm{MHz}$, such that the $72 \mathrm{nd}$ harmonic is at the desired synchronization frequency of $2.856 \mathrm{GHz}$. The laser is locked to the reference using a phase-locked loop (PLL), generating an error signal by comparing the 72nd harmonic of the 
laser repetition rate to the S-band reference. This is fed back to a fiber stretcher, onto which 2 meters of the laser cavity fiber are wound. By controlling the fiber length, the repetition rate is adjusted. The unity gain point of the PLL has to be chosen carefully to ensure that the final phase noise of the signal is as low as possible. In fact, the EDFL becomes the de facto master oscillator of the facility. By locking to an RF reference for better low frequency stability, the resulting phase noise of the laser combines the good low-frequency properties of the RF oscillator and the excellent high-frequency properties of the free-running EDFL. Hence, a net improvement of the phase noise is achieved.

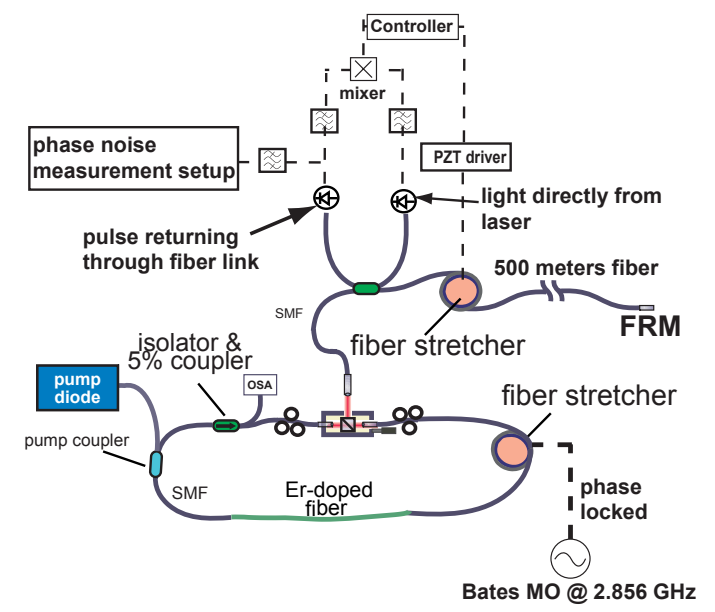

Figure 6. Schematic setup of the setup at MIT-Bates Laboratory.

\subsection{STABILIZATION OF THE FIBER LINK}

Optical fibers exhibit a temperature dependent change of the refractive index, which causes arrivel time jitter of a pulse propagating through the fiber. A typical value is $10^{-6} / \mathrm{C}^{\circ},{ }^{16}$ which corresponds to a fluctuation of 5 ps for a link length of $1 \mathrm{~km}$ and a temperature stability of $1 \mathrm{C}^{\circ}$. This makes a stabilization scheme mandatory. Presently, there are two different approaches to stabilize the fiber length, both relying on a fiber stretcher with sufficient stretch to adjust for length fluctuations. One approach is to interferometrically stabilize the link: light from a single-frequency laser is sent through the link and beat with the returning, frequency-shifted light, thus generating an RF frequency equal to the difference frequency of the optical waves. The phase error of the optical waves is directly transfered into the recovered RF frequency. This results in a very high resolution phase detector and faciliates the locking. ${ }^{17}$ However, this scheme stabilizes the phase velocity and not the group velocity. If any information is transmitted over the fiber link, it will travel with the group velocity. The difference between these two velocities is on the order of $10^{-2}$ and any temperature and stress dependent changes are difficult to control.

Stabilizing the fiber link using optical pulses has the inherent advantage of directly stabilizing the group velocity unlike schemes based on interferometric stabilization. Part of the light at the end of the link is reflected back into the same fiber using a Faraday rotator mirror. This mirror reflects the light with a 90 degree rotation of the polarization, counteracting the effects of residual birefringence in the fiber. There are two possible feedback schemes that can be employed. For a coarse lock, an RF-based scheme is used and optical crosscorrelation can be utilized for a fine lock. In the former scheme, part of the light directly from the laser and light returning through the fiber link are photodetected using two high-bandwidth photodiodes. Out of the resulting RF spectrum a harmonic is selected $(1 \mathrm{GHz}$, in the present study) and combined in quadrature in a mixer. The resulting phase error signal is fed back to the fiber stretcher. The mixer output is monitored with a high-resolution signal analyzer to assess the performance of the lock.

The results for both open and closed loop scenarios stabilizing the MIT-Bates fiber link mentioned above, is shown in Figure7. If the loop is open, the inloop jitter in a bandwidth between $0.1 \mathrm{~Hz}$ and $5 \mathrm{kHz}$ amounts 
to $66 \mathrm{fs}$, which is reduced to $12 \mathrm{fs}$ when the feedback is active. It should be emphasized that these results are by no means the limit achievable using this simple RF feedback approach. Using a higher harmonic determine the phase error in the mixer will lead to a linear increase in the resolution of the mixer. We expect to be able to decrease the residual timing jitter by 5-10 times if comparison frequencies in the 5-10 GHz range are used.

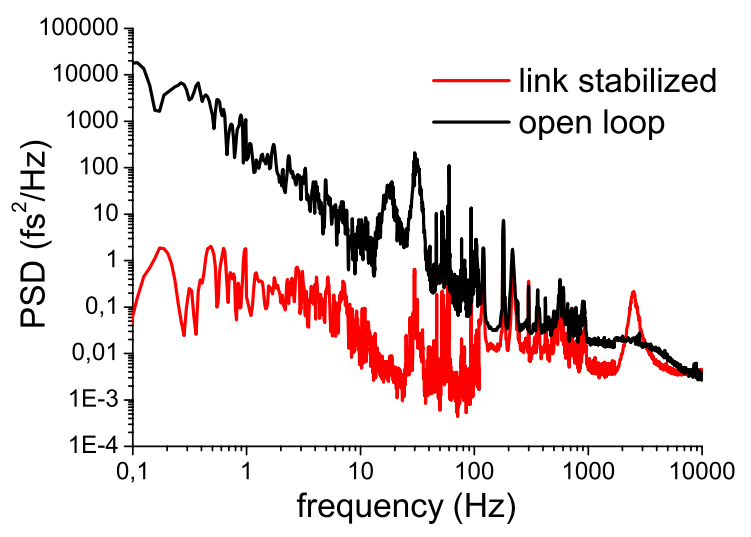

Figure 7. Mixer output signal of the RF fiber link stabilization.

We conclude that a fiber link of 500 meters length can readily be stabilized to the $10 \mathrm{fs}$ level using RF techniques alone. For a fine lock, it is possible to employ optical cross-correlation of the pulse coming directly from the laser with the one returning through the fiber. Here, the resolution depends predominantly on the pulse width used. For the proposed distribution system with a pulse length of several hundred femtoseconds, the resolution of the cross correlator can easily surpass the resolution achieved by an RF-based feedback by a factor of 100 and is comparable to the resolution of the interferometric stabilization scheme. If optical cross correlation is used as a second, additional feedback scheme, a stability level of below a femtosecond is feasible. ${ }^{18}$ Here, both pulses are overlapped either inside a crystal for second-harmonic generation or on a two-photon absorption detector. This results in a strong signal with a duration on the order of the pulse length of the incident pulses. This increases the resolution of this optical phase detector by a factor of 50 compared to the $\mathrm{RF}$ approach.

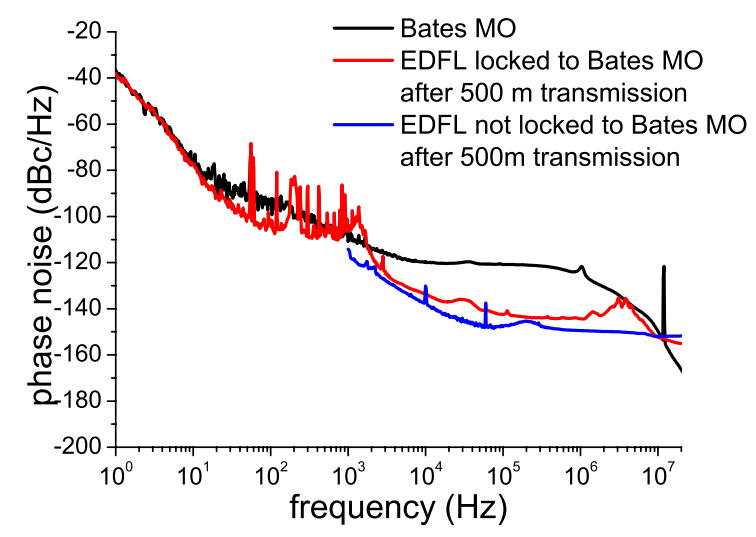

Figure 8. Single sideband phase noise of the Master Oscillator of the MIT-Bates Facility, the EDFL locked to the reference after transmission through the link and the free running EDFL. 


\subsection{RECOVERY OF THE RF SIGNAL AFTER TRANSMISSION}

The crucial question for any timing distribution system is the transmission of various RF signals with the required level of stability. After stabilizing the fiber link (500 m-long) and locking the EDFL to a local RF source, the phase noise of the signal at the end of the fiber link is the relevant parameter. Figure 8 shows the phase noise of the RF master oscillator at the MIT-Bates Facility and of the EDFL locked to the RF source after returning through the fiber link (total travel distance of $1 \mathrm{~km}$ ). The laser follows the RF master oscillator extremely well for the lower frequency range. For offset frequencies between $100 \mathrm{~Hz}$ and $10 \mathrm{kHz}$, there is technical noise added showing as spurs at $60 \mathrm{~Hz}$ and its harmonics. This is due to the driver of the pump diode and can be eliminated by running the pump diodes on battery power, or better isolation of the diode driver. At an offset frequency of $1.5 \mathrm{kHz}$, the free runnning spectra of the laser and the RF source meet, which corresponds to the optimal unity gain frequency for the PLL. As can be seen in Figure8, the locked EDFL phase noise spectrum follows the free running laser spectrum closely (blue line). The difference in these two is due to technical issues in the photodetection process, rather than additional noise due to the fiber transmission. The phase locking of the EDFL to the MIT-Bates master oscillator source adds around $30 \mathrm{fs}$ timing jitter. This is reduced to a few femtoseconds, if one ignores the technical noise spurs. The absolute phase noise is reduced, even taking the excess timing jitter due to the phase-lock into account, from 272 fs to 178 fs in a bandwidth of $10 \mathrm{~Hz}$ to $20 \mathrm{MHz}$. Including the residual noise of the fiber link stabilization, the complete system adds less than $50 \mathrm{fs}$ of timing jitter in a bandwidth of $0.1 \mathrm{~Hz}$ to $20 \mathrm{MHz}$.

\section{CONCLUSION AND OUTLOOK}

In conclusion, mode-locked lasers producing sub-ps pulses can serve as ultra-low noise master oscillators for timing distribution in next-generation light sources. The main advantage of mode-locked fiber laser is the excellent noise performance at high frequencies, the high quality and availability of pump sources and components in the $1550 \mathrm{~nm}$ wavelength range. Measurements show a high-frequency performance surpassing that of microwave oscillators with ultra-low phase noise. We have demonstrated sources with record low timing

jitter of $10 \mathrm{fs}$ in a bandwith of $1 \mathrm{kHz}$ to the Nyquist frequency. Such sources can be made readily available for sub-100 fs and potentially sub-50 fs timing distribution. We have demonstrated the operation of a complete timing distribution system consisting of the master laser oscillator locked to the S-band reference oscillator and one $500 \mathrm{~m}$-long stabilized fiber link. The residual inloop timing jitter due to the fiber link is $12 \mathrm{fs}$ rms between $0.1 \mathrm{~Hz}$ and $5 \mathrm{kHz}$ using an RF feedback system. Sub-fs timing jitter for the fiber link is feasible with optical cross correlation. The total added jitter due to locking of the laser to the microwave oscillator is $30 \mathrm{fs}$, leading to sub-50 fs of added jitter in a bandwidth of $0.1 \mathrm{~Hz}$ to $20 \mathrm{MHz}$. Overall, the way to a timing distribution system, with point-to-point jitter of $10 \mathrm{fs}$ is outlined. Finally, it should be noted that the initial transition from a laboratory implementation of this scheme to operation in a real-world accelerator environment has been remarkably smooth.

\section{REFERENCES}

1. M. Liepe, "Superconducting RF and RF Control" Proceedings of the ERL 2005 Workshop," Jefferson Lab, Newport News, VA.

2. S. Simrock, "Overview of options for RF control \& state of the art, simulation results and performance test results" Proceedings of the ERL 2005 workshop, Jefferson Lab, Newport News, VA.

3. A. Winter, F. Ö. Ilday, O. D. Mücke, R. Ell, H. Schlarb, P. Schmüser, and F. X. Kärtner, "Towards highperformance optical master oscillators for energy recovery linacs," Nuc. Instr. and Meth. A 557, 299-304 (2006).

4. J. W. Kim, F. Ö. Ilday, F. X. Kärtner, O. D. Mucke, M. H. Perrott, W. H. Graves, D. E. Moncton, and T. Zwart, "Large scale timing distribution and RF-synchronization for FEL facilities," FEL Conference 2004, Trieste, Italy, 2004.

5. J. Kim, F. X. Kärtner, and M. H. Perrott, "Femtosecond synchronization of radio frequency signals with optical pulse trains," Opt. Lett. 29, 2076-2078 (2004).

6. C. J. Boccetta et. al., "FERMI @ Elettra: A seeded harmonic cascade FEL for EUV and soft x-rays", Proceedings of the FEL 2005, Stanford, CA. 
7. A. Winter, E.-A. Knabbe, S. Simrock, B. Steffen, N. Ignachin, A. Simonov, S. Sytov, "Femtosecond synchronisation of ultrashort pulse lasers to a microwave RF clock," Proceedings of the PAC 2005, Knoxville TN.

8. F. Ö. Ilday, J. R. Buckley, H. Lim, and F. W. Wise, "Generation of 50-fs, 5-nJ pulses at $1.03 \mu \mathrm{m}$ from a wave-breaking-free fiber laser," Opt. Lett. 28, 1365-1367 (2003).

9. F. Ö. Ilday, J. R. Buckley, W. G. Clark, F. W. Wise, "Self-similar evolution of parabolic pulses in a laser," Phys. Rev. Lett. 92, 3902 (2004).

10. H. A. Haus, "Mode-locking of lasers," IEEE J. Sel. Top. Quantum Electron. 6, 1173-1185 (2000).

11. S. Namiki, and H. A. Haus, "Noise of the stretched pulse fiber ring laser: part I - theory," IEEE J. Quantum Electron. 33, 649-659 (1997).

12. C. X. Yu, S. Namiki, and H. A. Haus, "Noise of the stretched pulse fiber ring laser: part II - experiments," IEEE J. Quantum Electron. 33, 660-668 (1997).

13. J. P. Gordon, and H. A. Haus, "Random walk of coherently amplified solitons in optical fiber transmission," Opt. Lett. 11, 665-667 (1986).

14. K. Tamura, E. P. Ippen, H. A. Haus, and L. E. Nelson, "77-fs pulse generation from a stretched-pulse mode-locked all-fiber ring laser," Opt. Lett. 18, 1080-1082 (1993).

15. E. N. Ivanov, S. A. Diddams and L. Hollberg, "Analysis of noise mechanisms limiting the frequency stability of microwave signals generated with a femtosecond laser," IEEE J. Sel. Top. Quantum Electron. 9, 1059-1065 (2003).

16. C. E. Lee, H. F. Taylor, A. M. Markus, and E. Udd, "Optical-fiber Fabry-Perot embedded sensor," Opt. Lett. 14, 1225-1227 (1989).

17. J. W. Staples, and R. Wilcox, "Fiber transmission stabilization by optical heterodyning techniques and synchronization of mode-locked lasers using two spectral lines", Proceedings of the FEL 2005, Stanford, $\mathrm{CA}$, and references therein.

18. T. R. Schibli, J. Kim, O. Kuzucu, J. T. Gopinath, S. N. Tandon, G. S. Petrich, L. A. Kolodziejski, J. G. Fujimoto, E. P. Ippen, and F. X. Kärtner, "Attosecond active synchronization of passively mode-locked lasers by balanced cross correlation," Opt. Lett. 28, 947 (2003). 